\title{
Comparative analysis on datasets of myxomycetes associated with boreal, temperate and tropical regions of North America
}

\section{Carlos Rojas ${ }^{1 *}$ and Steven L. Stephenson ${ }^{2}$}

1 Engineering Research Institute and Department of Biosystems Engineering, University of Costa Rica, San Pedro de Montes de Oca, 11501-Costa Rica

2 Department of Biological Sciences, University of Arkansas, Fayetteville, Arkansas 72701

* Corresponding author:

carlos.rojasalvarado@ucr.ac.cr

Keywords: biogeography, biomes, datasets, macroecology, modelling, slime molds

Article info:

Received: 15 July 2020

Accepted: 11 September 2020

Published online: 13 October 2020

Corresponding Editor: Nikki Heherson A. Dagamac Assistant Editor: Oleg Shchepin

\begin{abstract}
Datasets from boreal (Denali National Park, United States), temperate (Great Smoky Mountains National Park, United States) and tropical (La Selva Biological Station, Costa Rica) regions of North America were subjected to analysis. The complete dataset, composed primarily of field data, consisted of 3558 records, with $46 \%$ temperate, $29 \%$ boreal and $23 \%$ tropical. A total of 208 species were recorded for the three regions, with $69 \%$ temperate, $49 \%$ boreal and $40 \%$ tropical. A high significant correlation between the number of records and the number of species $\left(r^{2}=0.99, P=0.001\right)$ suggested that the latter was a function of the former, independent of location. However, this relationship was stable at low survey efforts, as it was observed in a model obtained with 25 independent datasets from the northern hemisphere of the Americas. Diversity values, calculated with the Shannon Index, ranged from 3.4 to 4.0 and were different for all pairwise combinations (all cases $P<0.05)$ of the three datasets, but when calculat-
\end{abstract}


ed with the Simpson Index they were not different for the combination of temperate and boreal datasets. At the species level, the smallest value (0.38) for coefficient of community was observed for the boreal-tropical pair and highest (0.56) for the temperate-tropical pair. The taxonomic diversity indices were 2.68 and 2.83 for the boreal and tropical datasets, but 3.76 for the temperate dataset. The latter may be an indication of higher fruiting propensity in temperate regions rather than an indication of intraspecific diversity, an idea that deserves further examination. The boreal dataset had the highest number of unique genera (7), followed by the temperate (6) and the tropical (2) datasets. However, the temperate dataset showed the highest number of unique species (57), followed by the boreal (37) and tropical (26) datasets. When analyzed in a comparative context, standard experiments with similar field efforts and techniques are still required to document patterns of reproductive occurrence of myxomycetes in different regions of the world. For macroecological purposes, all regions represented by the datasets analyzed herein still remain understudied.

\section{Introduction}

The myxomycetes comprise a group of amoeboid protists known to occur in all biomes on the planet (Novozhilov et al. 2017b). Most of the large-scale spatial research on these organisms during the last 200 years has been based on the reproductive stage of their life cycle (Schnittler et al. 2017). Despite the incomplete information that has originated from such a shortcoming, particularly in terms of species absence, there is a large amount of data showing the opposite pattern (i.e., species presence).

In terms of species distribution and biogeographical patterns, the classical survey methodology used with myxomycetes has the intrinsic constraint of generating false negatives (i.e., falsely determining the absence of a species). However, it is also known that modern molecular methods, commonly used in the detection of microorganisms, have the constraint of generating false positives (i.e., falsely determining the presence of taxa by overes- timating biodiversity as noted by Guillera-Arroita [2016]). In this context, critical evaluations of mixed methodologies are necessary to design survey strategies that are less susceptible to the intrinsic biases of techniques and sampling.

Presence-only data, despite providing limited information about species distributions, are still remarkably important for ecological purposes (see Bradley 2016). With myxomycetes, it is clear that the accumulated information on their distribution, albeit incomplete due to the constraint explained earlier, is also limited by the fact that only one stage of their life cycle, the reproductive one, provides the information ("the tip of the iceberg" according to Schnittler et al. [2017]). However, those issues do not affect the robustness of data equally at all levels and apply mostly to biodiversity matters. In this sense, morphospecies-based, presence-only datasets still represent valuable tools for evaluation of the reproductive stage and for the study of ecological and evolutionary pressures directed on myxomycetes during the reproductive part of their life cycle.

In macroecological terms, such uncovering of fundamental relationships between species and the systems they inhabit at large spatial scales is important to understand the principles that determine, to a certain extent, their distribution (see Kent 2005). As such, examinations of potential ecological associations of species of myxomycetes, contribute to the knowledge of species distribution via species (or fruiting) occurrence. At large geographical scales, this is fundamental to examine biogeographical patterns and understand the basic drivers of myxomycete biodiversity (see Shoemaker et al. 2017). In simple terms, macroecological patterns can elucidate biogeographical ones.

One limitation, however, for comparative macroecological purposes using myxomycete data is the unavailability of reliable information for most ecosystems around the world. As such, available information on morphospecies presence is remarkably valuable within that context. During his career, the second author of this paper has generated a number of valuable datasets, including some in boreal, temperate, and tropical regions. Within that framework, the approach used herein was to examine one well-developed dataset in each one of three biomes in the northern hemisphere of the Americas. The 
objective of the latter was to analyze myxomycete occurrence in the three regions, using data for the reproductive stage of their life cycle, for a comparison of results that can contribute to the creation of a representative picture of the biological complexity present in them. This is an important task for the elucidation of macroecological dynamics than can potentially serve as the basis of monitoring.

\section{Material and Methods}

Three datasets, obtained from the database of worldwide myxomycete records at the University of Arkansas, were used for this study. All of them were selected on the basis of geographical location (i.e., representative of a particular biome) and the number of records contained. In this manner, datasets from Denali National Park (DNP hereafter, $63^{\circ} \mathrm{N}$, Alaska, USA), the Great Smoky Mountains National Park (GSMNP, $35^{\circ} \mathrm{N}$, Tennessee/North Carolina,
USA) and the La Selva Biological Station (LSBS, $10^{\circ} \mathrm{N}$, Costa Rica) were selected for the characterization of boreal (taiga), seasonal temperate and tropical rainforests, respectively. These three locations represented an extreme gradient of naturally occurring biomes, each one separated between $25^{\circ}-28^{\circ}$ of latitude, in the northern hemisphere of the Americas.

After correcting inconsistencies in the datasets (i.e., old names, records with incomplete information, and clearly wrong data), a calculation of alpha diversity for each one was carried out using both the Shannon's and the Simpson's diversity indices. Also, the Taxonomic Diversity Index (number of species/number of genera) was calculated and the diversity profile for each set was also constructed using Hill numbers. The Shannon Index was calculated for reference since a number of previous studies on myxomycetes have calculated this estimator; however, due its difficult interpretation, the intuitive form of the Simpson's Index (1-D) was calculated as well. The diversity profiles in the sense of Chao et al (2014) represent a more accurate strategy for interpretation of alpha diversity dynamics and in

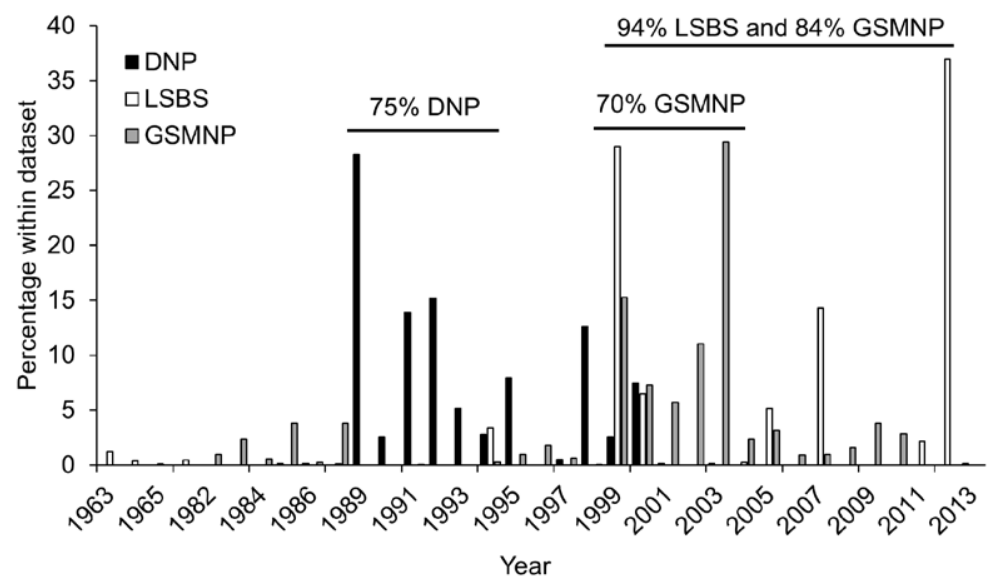

Figure 1. Relative effort of myxomycete recording by time in the three datasets studied herein. Temporal sections with high collecting effort have been marked. 
Table 1. Diversity estimators (and range) calculated for the three datasets analyzed in the present study. Highest values are shown in bold. The abbreviations for the datasets are provided in the Material and Methods.

\begin{tabular}{llll}
\hline & Datasets & & \\
& DNP & GSMNP & LSBS \\
\hline Shannon's Diversity Index & $3.90(3.88-3.98)$ & $\mathbf{4 . 0 5}(4.08-4.19)$ & $3.43(3.37-3.53)$ \\
Simpson's Diversity Index & $\mathbf{0 . 9 7}(0.96-0.97)$ & $0.96(0.95-0.97)$ & $0.94(0.93-0.95)$ \\
Chao 1 - Maximum Number of species & $137(110-140)$ & $\mathbf{1 7 2}(150-180)$ & $128(90-131)$ \\
Taxonomic Diversity Index & 2.68 & $\mathbf{3 . 7 6}$ & 2.83 \\
\hline
\end{tabular}

these, a Hill number $q=0$ is associated with species richness, $q=1$ indicates the number of species with typical abundances, and $q=2$ refers to the effective number of dominant species.

A general rarefaction curve was created for each dataset for comparison purposes. With these curves, the relative accumulation of species can be compared between different sets at the same sampling effort. Similarly, a correlation between the number of records and the number of species was carried out to test the hypothesis that the relationship between the two variables is independent of location. With the use of 25 datasets of myxomycete records from North America (obtained from Lado \& Rojas [2018] and Stephenson et al. [2020]), a general model was created to assess the last idea in a broader context and to evaluate the position of the three studied datasets in relation with the general trend of species accumulation.

For beta diversity analysis, two approaches were also followed. The commonly used Sørensen coefficient of community estimator was calculated for all pairwise combinations and a cluster analysis using the Jaccard algorithm was carried out with the complete three datasets. The latter followed the "regional" approach discussed by Chao and Chun-
Huo (2016). In both cases, the number of records associated with the different species was used for the calculations (and also as a proxy for species abundance). In a similar manner, the unique number of species and genera was calculated for each dataset and the number of shared taxa between two datasets were determined. The effective proportion of nonshared species in the pooled community was calculated. A second cluster analysis with the data at the genus level was also carried out. The beta diversity approach is intended to create a picture of the ratio between regional and local diversity. Following the observations of Sreekar et al. (2018), a null hypothesis of no differences in beta diversity due to the large spatial scaling was established for all three locations. For the latter, the shared Jaccard estimator of beta diversity was used in the model as a reference of comparison and a Wilcoxon test was used.

All diversity-based calculations were carried out using the SpadeR program in R (Chao et al. 2016) and statistical analyses were performed in PAST v4.01 (Hammer et al. 2001). In all cases, the cutoff value to accept the null hypothesis was 0.05 . The nomenclature was established according to Lado (2005-2020). 


\section{Results}

The complete dataset contained 3558 records of myxomycetes with 1684 records from GSMNP (40\%), 1043 from DNP (29\%) and 831 (23\%) from LSBS. All records were obtained between 1963 and 2013 (Fig. 1). The collecting effort in the different locations took place at different times with about $75 \%$ of all records from DNP made between 1989 and 1995 (7 year period), 70\% of records from the GSMNP made between 1999 and 2003 (5 year period) and about $94 \%$ of records from LSBS made between 1999 and 2012 (14 year period).
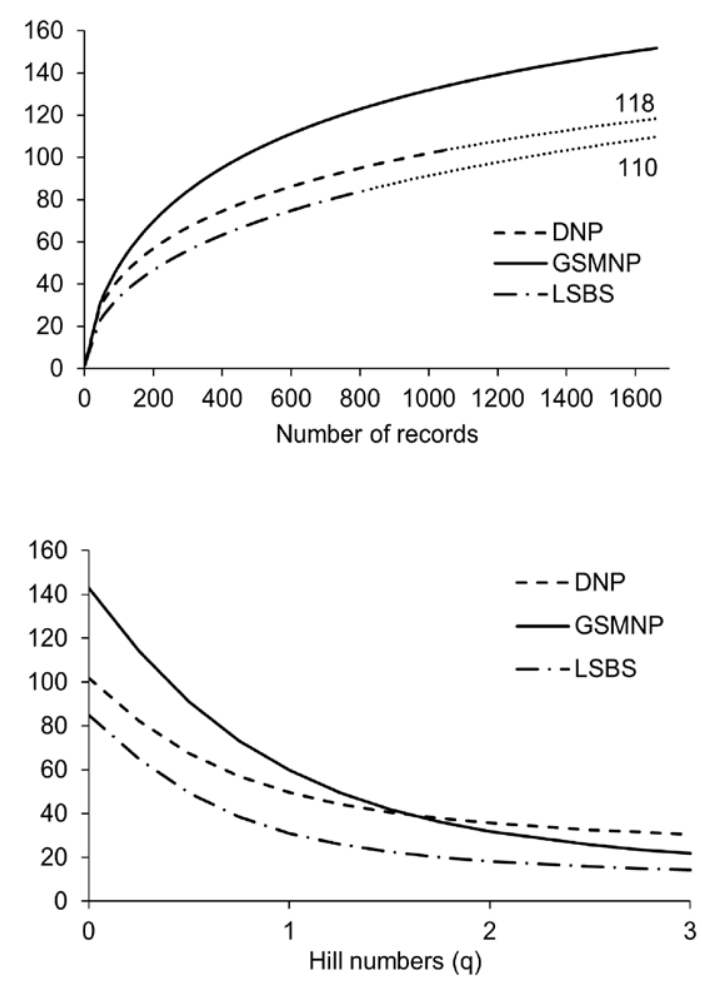

Figure 2. Rarefaction (top) and diversity profile (bottom) curves constructed with the three datasets studied herein. For the first one, dotted sections represent extrapolated values. For interpretation of $q$ values see the Materials and Methods section.
A total of 143 species were recorded in GSMNP, followed by 102 from DNP and 85 from LSBS. Using the number of records as a proxy for collecting effort, there was a high significant correlation between effort and the number of species recorded $\left(r^{2}=0.99\right.$, $P=0.001)$. The highest Shannon Index of Diversity was observed in GSMNP, followed by DNP and LSBS (Table 1) and all combinations were significantly different $(P<0.05$ in all cases). The highest Simpson Index of Diversity was calculated for DNP, followed by GSMNP and LSBS and the pairwise comparison of diversity values between GSMNP and DNP was not significant. The highest Taxonomic Diversity Index was observed in GSMNP, followed by LSBS and DNP.

The rarefaction curves constructed with the data (Fig. 2, top) showed that the lowest number of maximum expected species was associated with LSBS with approximately 110 species. The GSMNP was associated, as expected, with the highest values of accumulated recorded species based on a similar effort. For this dataset, however, the diversity profiles (Fig. 2, bottom) showed a lower number of species with typical abundances and dominant species ( $q=2$ and 3 ) than DNP. The LSBS dataset showed the lowest values at all Hill number values.

The values of records and species observed in the three datasets studied herein placed them in the growing part of the curve of species accumulation constructed with 25 datasets from the northern hemisphere of The Americas (Fig. 3). Such a model showed a remarkably high correlation value $(\mathrm{r}=0.97)$ and a classical shape produced by equation 1 .

$$
y=\frac{822.7+330.9 \times x^{0.73}}{227.9+X^{0.73}}
$$

(equation 1)

Where $x$ is the number or records of myxomycetes in a dataset and 330.9 indicates the maximum number of species to be recorded (the asymptote) in a dataset at the maximum spatial scale studied herein (about 80000 square kilometers)

The average deviation of the actual observed values obtained from the datasets and the model was $-1.3 \times 10^{-8}$ units (species, $y$ axis) but the average 


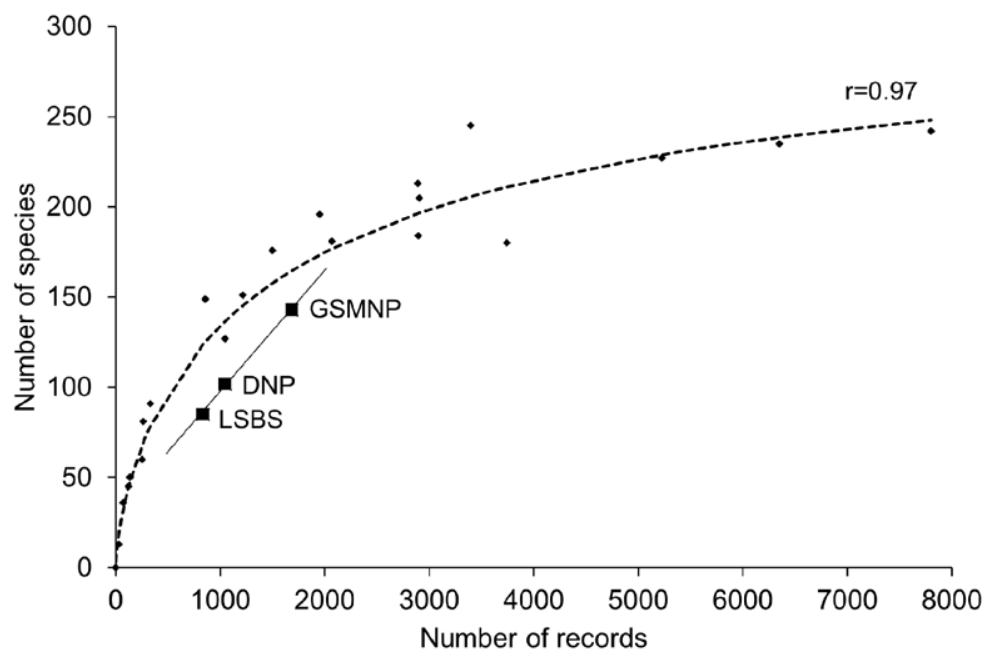

Figure 3. Model of species accumulation as a function of number of records observed for 15 independent datasets from North America (dotted line, high correlation) and real observed values (markers). The three datasets studied herein are shown in the graph along with the linear relationship among them (solid line, $r=0.99$ ).

deviation obtained for the three datasets studied herein was -31.5 units. This suggests that all three datasets are underestimating the number of species at their respective effort based on a general trend.

When the beta diversity analyses were carried out, results showed that, at the species level, the overall similarity among the three datasets was 0.29 (range between 0.26-0.32) when a classical species richness-based regional estimator such as Jaccard was used. With this approach, the most dissimilar dataset was DNP (Fig. 4, left) but no significant differences were found $(W=5, P=0.28)$ in similarity across datasets. The coefficient of community values, which are focused more on local diversity (Fig. 4, left) showed a similar pattern, with an average of 0.55 (range between 0.51-0.59) and highest differences between DNP and LSBS.

When a similar evaluation was performed at the genus level, a different pattern was observed in the cluster analysis (Fig. 4, right). In this case, the most dissimilar dataset was LSBS. However, differences in the values of the Jaccard estimator as well as the coefficient of community were very small, as anticipated at a larger taxonomic scale. Since most genera where shared among datasets (22 in average or $45 \%$ of total number of genera) differences were smaller than at the species level.

With a total of 63 shared species, the pair DNPGSMNP showed the highest value of taxonomic overlap. This was followed by 57 shared species between GSMPN-LSBS and only 36 between DNPLSBS. The latter was similar to the 34 shared species across the three datasets, including Ceratiomyxa fruticulosa, Lycogala epidendrum and Stemonitis axifera. At the same time, GSMNP showed 57 unique species including Colloderma oculatum, Trichia subfusca and Lamproderma columbinum. This was followed by the 37 unique species in DNP, which included Mucilago crustacea, Trichia flavicoma and Craterium leucocephalum; and 26 unique species in LSBS, including Arcyria afroalpina, Physarella oblonga and Physarum melleum. Interestingly, the effective proportion of non-shared species in the pooled data from all three datasets was calculated in $56 \%$ of the species. At the genus level, DNP showed seven unique genera, with Calomyxa and Oligonema among them; GSMNP had six unique genera, with Barbeyella and Colloderma, and only Physarella and Stemonaria were unique to LSBS (see Table 2) 
Jaccard Similarity (Species level)

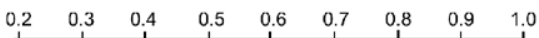
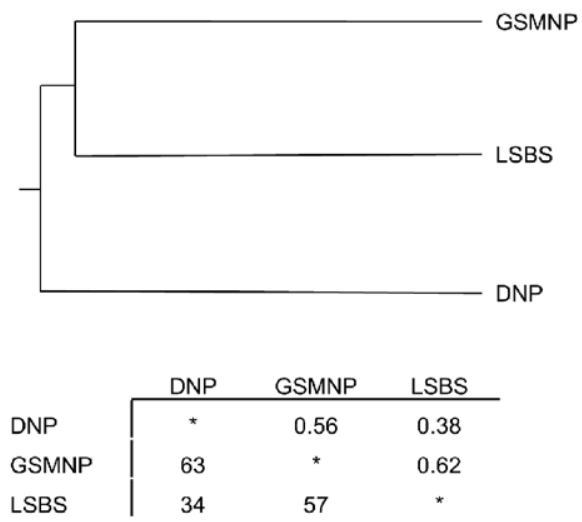

Jaccard Similarity (Genus level)

$\begin{array}{lllllllll}0.55 & 0.60 & 0.65 & 0.70 & 0.75 & 0.80 & 0.85 & 0.90 & 0.95\end{array}$

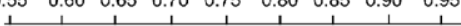

Figure 4. Diagram of the relationships between the studied datasets using records of myxomycete species (left) and genera (right) and the Jaccard similarity as an estimator of beta diversity (top). The matrices (bottom) show the coefficient of community values observed for pairwise combinations (above) and the number of shared taxa (below). The abbreviations of the datasets are provided in Material and Methods.

\section{Discussion}

This study was not intended to analyze myxomycete biodiversity and biogeography. As pointed out by Schnittler et al. (2017), those are topics for which molecular information would facilitate a robust examination of patterns. However, as mentioned before, the current available datasets on myxomycete occurrence (field-based presence only data) are remarkably valuable for ecological analysis. In that manner, the purpose of this study was to examine myxomycete presence in three biomes of the world, to contribute, with comparative analyses, to the elucidation of macroecological patterns. Even though the datasets presented herein are primarily, but not entirely, generated with field records, it is important to note that an even higher level of representativeness could be accomplished in an analysis made with a completely known nature of records.

It is interesting to note that the shortest period of time associated with recording information for the datasets considered in the present study was that in the Great Smoky Mountains National
Park. However, it makes perfect sense since the period between 1997-2003 was a very active time for biological research at that park. It was the peak of the All Taxa Biodiversity Inventory initiative (see Sharkey 2001), a project designed to document the biodiversity of a number of biological groups in the area. Both the datasets at Denali National Park and La Selva Biological Station followed a grant-based model of generation of information, with which data accumulated at a slower pace. In this sense, the datasets studied in the present investigation demonstrated that an increased effort of myxomycete recording in one general location had a positive effect in the faster accumulation of data.

Despite the latter, the high linear correlation of species accumulation and records (effort) observed herein, in the context of the general model constructed with independent data, clearly showed that neither of the three datasets can be considered a complete picture of field-based information on myxomycetes for their respective biome. However, those results also showed that in terms of stimulus-response at the 
Table 2. Number of records of myxomycetes, arranged by genus, associated with the three datasets studied in the present investigation. Numbers in bold denote unique genera for particular datasets.

\begin{tabular}{|c|c|c|c|c|c|c|c|}
\hline \multirow[t]{2}{*}{ Genus } & \multicolumn{3}{|c|}{ Dataset } & \multirow[t]{2}{*}{ Genus } & \multicolumn{3}{|c|}{ Dataset } \\
\hline & DNP & GSMNP & LSBS & & DNP & GSMNP & LSBS \\
\hline Arcyodes & 3 & 1 & & Hemitrichia & 58 & 85 & 63 \\
\hline Arcyria & 71 & 241 & 153 & Lamproderma & 4 & 30 & 26 \\
\hline Badhamia & 6 & 6 & & Leocarpus & 37 & 16 & \\
\hline Badhamiopsis & & 3 & & Lepidoderma & 1 & 17 & \\
\hline Barbeyella & & 11 & & Licea & 15 & 22 & 3 \\
\hline Calomyxa & 11 & & & Lindbladia & & 1 & \\
\hline Calonema & & & 1 & Lycogala & 61 & 49 & 14 \\
\hline Ceratiomyxa & 33 & 42 & 76 & Macbrideola & 2 & & \\
\hline Clastoderma & 1 & 10 & 2 & Metatrichia & 6 & 22 & 2 \\
\hline Collaria & 1 & 32 & 6 & Mucilago & 47 & & \\
\hline Colloderma & & 19 & & Oligonema & 2 & & \\
\hline Comatricha & 27 & 42 & 14 & Paradiacheopsis & 1 & & \\
\hline Craterium & 18 & 11 & 2 & Perichaena & 89 & 35 & 34 \\
\hline Cribraria & 22 & 141 & 41 & Physarella & & & 10 \\
\hline Diachea & & 3 & 1 & Physarum & 173 & 325 & 193 \\
\hline Diacheopsis & & 3 & & Reticularia & 2 & 1 & \\
\hline Dianema & 1 & 3 & & Siphoptychium & 3 & & 1 \\
\hline Dictydiaethalium & & 4 & 2 & Stemonaria & 1 & & 1 \\
\hline Diderma & 17 & 44 & 37 & Stemonitis & 58 & 73 & 22 \\
\hline Didymium & 36 & 99 & 111 & Stemonitopsis & 10 & 42 & 4 \\
\hline Echinostelium & 10 & 8 & 4 & Symphytocarpus & & 1 & 1 \\
\hline Elaeomyxa & & 8 & & Trichia & 155 & 163 & 4 \\
\hline Enerthenema & 6 & 10 & & Tubifera & 4 & 34 & 2 \\
\hline Enteridium & 1 & & & Willkommlangea & 1 & & \\
\hline Fuligo & 47 & 18 & 1 & & & & \\
\hline
\end{tabular}


reproductive stage, all biomes are essentially providing equivalent conditions for myxomycetes to thrive. Such neutrality in the boreal-temperate-tropical gradient has been observed in plant responses to the environment (Bastias et al. 2017) and would deserve a finer exploration with other myxomycete data. In this sense, differences in myxomycete reproductive dynamics according to biome are more related with the length of growing seasons than with the biological quality of the systems (in the sense of Cavahaugh et al. 2014). It is clear that the length of the season with such conditions (i.e., non-winter months) follows the pattern $\mathrm{DNP}<\mathrm{GSMNP}<\mathrm{LSBS}$ and that myxomycetes enter the stage of formation of reproductive structures during that time. As such, and as many myxomycete researchers would know, the accumulation of new species using field methods is slower in tropical areas (see Novozhilov et al. [2017a] for Vietnam, Lado \& Rojas [2018] for Costa Rica), which can partially be attributed to the extremely long (comparatively) season with favorable conditions. In these areas, there are always fruiting bodies of myxomycetes in the field, but very often they tend to represent a core of extremely common species. The diversity indices calculated herein partially demonstrated the latter, which was clearer to observe in both the rarefaction curve and the diversity profiles.

One aspect that was interesting to observe is that both the Simpson's Index of Diversity (which focuses on dominance) and the Hill number (q) of order 3 (also focusing on dominance) showed higher values associated with DNP than any other dataset. These results referred to the fact that in DNP there was a higher number of dominant species and not necessarily a few extremely dominant ones. In other words, the DNP dataset was not the richest in species but was structured by tiers of incidental species (one of which showed dominance) that responded more or less similarly to others within the same tier. This result suggested that the shortest but highly favorable period for production of fruiting bodies in DNP, in which other elements of the ecosystem are also active, was detected with myxomycete data. Basically, as different components of the system become active after long winters, myxomycete activity is stimulated as well, facilitating a more homogeneous production of fruiting bodies associated with those different ecological components.
Such a phenomenon, in a way, is also present in temperate forests. However, the higher number of compartments within the ecosystem in this biome (compared to the boreal one) allows for a potentially higher number of niches to be occupied. The Great Smoky Mountains have been known to be a good example of biological complexity in temperate areas for a long time (see Whittaker 1956). If, by mere probability, some species of myxomycete occupying these niches would also fruit during the favorable seasons after the winter, a higher number of species would be expected to be recorded. The higher Taxonomic Diversity Index and number of unique species associated with the GSMNP dataset may be an indication of such higher fruiting propensity in temperate regions rather than an indication of intraspecific or overall diversity. In a similar manner to what has been observed with some fungi (McGuire et al. 2013), there are differences in taxonomic composition of species among biomes (via recording of fruiting bodies) but very analogous underlying ecological patterns shaping the visible stage of the species. Again, the equivalency of data accumulation across the three datasets studied herein points in that direction and supports the high correlation found in a general model. Those results are also supported by the beta-diversity analysis where no differences were observed across the three datasets.

Again, differences were obviously recorded in the species composition associated with each biome. Some species and even genera were observed only in one dataset, as expected. This would simply reflect the long known association of taxa with specific environments (see Schnittler et al. [2017], for Barbeyella). However, the results obtained herein suggest, as explained by Sreekar et al. (2018), that at large scales, differences in gradient overlap are more stable than previously acknowledged. With the myxomycete data analyzed in the present study, such pattern could be reflected in results only if there is a similar probability of production of fruiting bodies across biomes, which as discussed earlier, seems to be the case during the period with favorable conditions. Myxomycete occurrence (again, via recording of fruiting bodies) seems to be more affected by substrate availability and microenvironmental conditions (see Schnittler et al. [2016] for corticolous myxomycetes in Alaska) than by the general char- 
acteristics of the biome. As long as the ecosystem creates potential niches for the fruiting bodies to form and environmental conditions are favorable, different species would enter their reproductive stage and effectively form these structures.

The present study has been designed using well-developed datasets (for myxomycete standards) for the respective biomes and has shown that for practical purposes, all three systems are understudied. More importantly, this investigation has demonstrated that macroecological considerations across biomes are still poorly documented, albeit the clear possibilities facilitated by the available data. Of course, several other datasets have been generated in recent decades for the same biomes (i.e., Novozhilov et al. [1999] in boreal areas, Schnittler et al. [2006] for temperate ones, Stephenson et al. [2004] for tropical ones) and they are important for biodiversity and ecosystem/microhabitat studies. However, for analyses at a macroscale (e.g., macroecology or climate change), long-term datasets generated by an intense recording effort in single areas are essential. Simon Levin (1992) pointed out that "the problem of pattern and scale is the central problem in ecology... there is no single natural scale at which ecological phenomena should be studied; systems generally show characteristic variability on a range of spatial, temporal, and organizational scales". In this sense, myxomycete ecology and biogeography is still highly understudied, not only at different scales but also in relation with patterns of detection beyond biodiversity-related matters.

\section{Acknowledgements}

Appreciation is extended to Richard W. Stauffacher for the generation of two of the datasets used in the present study. A large number of records considered in this investigation were obtained through research grants to SLS, including support from the National Geographic Society, the National Science Foundation, and Discover Life in America. Additional support was received from Universidad de Costa Rica through Vicerrectoría de Investigación (570-B8-006).

\section{References}

Bastias, C.C., Fortunel, C., Valladares, F., Baraloto, C., Benavides, R., Cornwell, W., Markesteijn, L., de Oliveira, A.A., Sansevero, J.B.B., Vaz, M.C. \& Kraft, N.J.B. 2017. Intraspecific leaf trait variability along a boreal-to-tropical community diversity gradient. PLOS ONE 12: e0172495.

Bradley, B.A. 2016. Predicting abundance with presence-only models. Landscape Ecology 31: 19-30.

Cavanaugh, K.C., Gosnell, J.S., Davis, S.L., Ahumada, J., Boundja, P., Clark, D.B., Mugerwa, B., Jansen, P.A., O'Brien, T.G., Rovero, F., Sheil, D., Vasquez, R. \& Andelman, S. 2014. Carbon storage in tropical forests correlates with taxonomic diversity and functional dominance on a global scale. Global Ecology and Biogeography 23: 563-573.

Chao, A., Gotelli, N., Hsieh, T.C., Sander, E., Ma, K., Colwell, R. \& Ellison A. 2014. Rarefaction and extrapolation with Hill numbers: A framework for sampling and estimation in species diversity studies. Ecological Monographs 84: 45-67.

Chao, A. \& Chun-Huo, C. 2016. Bridging the variance and diversity decomposition approaches to beta diversity via similarity and differentiation measures. Methods in Ecology and Evolution 7: 919-928.

Chao, A., Ma, K.H., Hsieh, T.C. \& Chiu, C.H. 2016. SpadeR (Species-richness Prediction And Diversity Estimation in R): an R package in CRAN. https://cran.r-project.org/web/ packages/SpadeR/index.html (15 June, 2020).

Guillera-Arroita, G. 2016. Modelling of species distributions, range dynamics and communities under imperfect detection: advances, challenges and opportunities. Ecography 40: 281-291. 
Hammer, Ø., Harper, D.A.T., Ryan, P.D. 2001. PAST: Paleontological Statistics software package for education and data analysis. Palaeontologia Electronica 4: 1-9.

Kent, M. 2005. Biogeography and macroecology. Progress in Physical Geography 29: 256-264.

Levin, S. 1992. The Problem of Pattern and Scale in Ecology: The Robert H. MacArthur Award Lecture. Ecology 73: 1943-1967.

Lado, C. 2005-2020. An on line nomenclatural information system of Eumycetozoa. Real Jardín Botánico, CSIC. Madrid, Spain. http://www.nomen.eumycetozoa.com (4 July 2020).

Lado, C. \& Rojas, C. 2018. Diversity patterns, ecological associations and future of research on Costa Rican myxomycetes. Mycology 9: 250-263.

McGuire, K.L., Allison, S.D., Fierer, N. \& Treseder, K.K. 2013. Ectomycorrhizal-Dominated Boreal and Tropical Forests Have Distinct Fungal Communities, but Analogous Spatial Patterns across Soil Horizons. PLOS ONE 8: e68278.

Novozhilov, Y.K., Erastova, D.A., Shchepin, O.N., Schnittler, M., Alexandrova, A.V., Popov, E.S. \& Kuznetov, A.N. 2017a.

Myxomycetes associated with monsoon lowland tropical forests in southern Vietnam. Nova Hedwigia 104: 143-182.

Novozhilov, Y.K., Rollins, A.W. \& Schnittler, M. 2017b. Ecology and Distribution of Myxomycetes. In: Stephenson, S.L. \& Rojas, C. (eds.). Myxomycetes: Biology, Systematics, Biogeography and Ecology, 253-297. Academic Press, London.

Novozhilov, Y.K., Schnittler, M., \& Stephenson, S.L. 1999.

Myxomycetes of the Taimyr Peninsula (North-Central Siberia). Karstenia 39: 77-97.

Schnittler, M., Dagamac, N.H.A., Sauke, M., Wilmking, M., Buras, A., Ahlgrimm, S., \& Eusemann, P. 2016. Ecological factors limiting occurrence of corticolous myxomycetes a case study from Alaska. Fungal Ecology 21: 16-23.

Schnittler, M., Dagamac N.A.H. \& Novozhilov, Y. 2017. Biogeographical patterns in Myxomycetes. In: Stephenson, S.L. \& Rojas, C. (eds.). Myxomycetes: Biology, Systematics, Biogeography and Ecology, 299-331. Academic Press, London.

Schnittler, M., Unterseher, M. \& Tesmer, J. 2006. Species richness and ecological characterization of myxomycetes and myxomycete-like organisms in the canopy of a temperate deciduous forest. Mycologia: 98:223-32.
Sharkey, M.J. 2001. The All Taxa Biological Inventory of the Great Smoky Mountains National Park. The Florida Entomologist 84: 556-564.

Shoemaker, W.R., Locey, K.J. \& Lennon, J.T. 2017. A macroecological theory of microbial biodiversity. Nature Ecology and Evolution 1: 0107.

Stephenson, S.L., Schnittler, M. \& Lado, C. 2004. Ecological characterization of a tropical myxomycete assemblage-Maquipucuna Cloud Forest Reserve, Ecuador. Mycologia 96: 488-497.

Stephenson, S.L., Stauffacher, R.W, \& Rojas, C. 2020. Myxomycetes collected in the eastern United States and patterns of relative biodiversity. Mycology. http://doi.org/10.1080/21501203.2019.1710302

Sreekar, R., Katabuchi, M., Nakamura, A., Corlett, R.T., Slik, J.W.F., Fletcher, C., He, F., Weiblen, G.D., Shen, G., Xu, H., Sun, I.F., Cao, K., Ma, K., Chang, L.W., Cao, M., Jiang, M., Gunatilleke, I.A.U.N., Ong, P., Yap, S., Gunatilleke, C.V.S., Novotny, V., Brockelman, W.Y., Xiang, W., Mi, X., Li, X., Wang, X., Qiao, X., Li, Y., Tan, S., Condit, R., Harrison, R.D. \& Koh, L.P. 2018. Spatial scale changes the relationship between beta diversity, species richness and latitude. Royal Society Open Science 5:181168.

Whittaker, R.H. 1956. Vegetation of the Great Smoky Mountains. Ecological Monographs 26: 1-80. 